\title{
Foot care education and self management behaviors in diverse veterans with diabetes
}

\author{
Jonathan M Olson' \\ Molly T Hogan ${ }^{2}$ \\ Leonard M Pogach ${ }^{3}$ \\ Mangala Rajan ${ }^{3}$ \\ Gregory J Raugi ${ }^{4}$ \\ Gayle E Reiber ${ }^{5}$
}

'University of Washington School of Medicine, Seattle, WA, USA; ${ }^{2}$ Department of Internal Medicine, University of Washington School of Medicine, Seattle, WA, USA; ${ }^{3}$ Department of Veterans Affairs, New Jersey Healthcare System, Center for Healthcare Knowledge Management, East Orange, NJ, USA; ${ }^{4}$ Division of Dermatology, VA Puget Sound Healthcare System, Department of Veterans Affairs, Seattle, WA, USA; ${ }^{5}$ Research and Development, VA Puget Sound Healthcare System, Department of Veterans Affairs, Seattle, WA, USA

Correspondence: Gayle Reiber VA HSR\&D, I 100 Olive Way, Ste 1400, Seattle,WA 9810।, USA

Tel +l 2067642089

Fax +I 2067642935

Email greiber@u.washington.edu

\begin{abstract}
The objective of this study was to examine differences in self-reported diabetes foot care education, self management behaviors, and barriers to good foot care among veterans with diabetes by race and ethnicity. Data was collected using the Veterans Health Administration Footcare Survey, a validated tool that assessed demographic, general health, diabetes and foot self-care information, barriers to foot self-care, receipt of professional foot care, and satisfaction with current care. We mailed surveys to a random sample of patients with diabetes from eight VA medical centers. Study participants were 81\% White; 13\% African American; 4\% Asian, and 2\% American Indian and Pacific Islanders. The majority of respondents felt that they did not know enough about foot self-care. There were large gaps between self-reported knowledge and actual foot care practices, even among those who reported "knowing enough" on a given topic. There were significant differences in self-reported foot care behaviors and education by race and ethnicity. These findings document the need for culturally-specific self-management education to address unique cultural preferences and barriers to care.
\end{abstract}

Keywords: diabetes mellitus, diabetic foot, patient self-management, ethnic groups, education

\section{Introduction}

Diabetes is a prevalent chronic condition, affecting $8 \%$ of the US population, with a disproportionate impact by race and ethnicity. The age-adjusted 2007 diabetes prevalence among African Americans was 11.8\%; among Hispanics 10.4\%; and among Whites 6.6\%. ${ }^{1}$ The Department of Veterans Affairs (VA) manages the largest health care organization in the US. Among veterans receiving VA care, $20 \%$ are diagnosed with diabetes, ${ }^{2}$ approximately $24 \%$ are non-White, ${ }^{3}$ and over half have risk factors for lower-limb ulcers and amputations. ${ }^{4,5}$ Although non-White veterans have documented disparities in the quality of some diabetes care processes and intermediate outcome measures ${ }^{6}$ racial disparities in foot care have received little attention.

The National Behavioral Risk Factor Surveillance System identified that $65 \%$ of veterans using VA services received education, compared to $47 \%$ of veterans who did not use VA services, yet many veterans lack the self-management skills needed to manage their high-risk foot conditions. ${ }^{3,7}$ Education about diabetes self-management involves issues of knowledge, preferences, culture, and unique barriers to care. ${ }^{8,9}$

Most studies examining diabetes self-management ${ }^{10-12}$ have not examined differences along racial and ethnic lines. In studies that have stratified by race, few have examined foot care. Therefore, the purpose of this study was to examine veterans' self-reported foot care education, current self-management behavior, and barriers to good foot care by race as defined by the census for US veterans receiving VA care.

\section{Conceptual framework}

This research builds on self-efficacy and self-management theory: the ability to plan and take action to reach a particular goal. Self-efficacy strategies have been shown to 
improve health outcomes in patients with chronic diseases including arthritis, lung disease, heart disease, stroke, ${ }^{13}$ and diabetes. ${ }^{14}$ Though there has been some success with glycemic control using a self-efficacy model, ${ }^{8}$ it remains difficult to translate self-efficacy theory into real-world behaviors, particularly in diabetic patients. ${ }^{15}$ Nwasuruba and colleagues ${ }^{16}$ report a profound disconnect between perceived knowledge and ability to implement self-management behaviors in diabetic patients, with regard to physical activity, diet, glucose testing, and foot care. In the VA, the disparity is heightened among minority populations, ${ }^{6}$ where there is a large gap between the theory and practice of selfmanagement. In addition, culture and attitudes surrounding chronic disease may differ sharply along racial and ethnic lines ${ }^{17}$ and need to be considered when implementing a successful model of care.

\section{Methods}

\section{Survey description}

The Veterans Health Administration Footcare Survey was developed in 2001 and administered in eight VA medical centers after obtaining Human Subjects approval. ${ }^{7,18}$ Questions on health transitions, physical function, and overall health were derived from the SF-36 and the Medical Outcomes Study. ${ }^{19}$ Relevant questions on foot-risk factors, self-care behaviors and education were derived from the Diabetes Quality Improvement Project Survey ${ }^{20}$ and supplemented by expert opinion. Study team members, including clinical foot care experts, survey design experts, and psychometricians then reviewed items for structure and clarity before pilot testing the questionnaire with patient focus groups. The final, machine-readable survey covers demographic, general health and diabetes, diabetes education and foot self-care information. ${ }^{7}$ The questions also probed barriers to foot selfcare, receipt of professional foot care, specialized footwear and satisfaction with current foot care.

\section{Measures}

Multiple dimensions of behavior were self-reported including foot self-care, footwear use, and foot care-seeking behaviors. In addition, a series of questions asked about foot care provided by clinicians, eg, callus care. Foot care education questions were introduced using the framing question, "How much have you ever been taught about taking care of your feet?" Responses were scored on a 4-point ordinal scale where $1=$ Nothing at all; 2 = A little bit; 3 = Some, but would like to know more; and 4 = Enough. The responses "enough" and "nothing at all" were selected from the questions regarding self-reported foot care education (Table 2). Race was grouped into four categories: White (including Hispanic), African American, Asian, and American Indian/Pacific Islander.

\section{Sampling and data collection}

A diabetes registry for veterans at each of the eight facilities was constructed using HEDIS criteria for diabetes ( $\geq 2$ outpatient contacts or at least one inpatient admission with a diabetes-specific ICD-9 code: 250.0-250.9, 357.2, $362.0,362.01,362.02$, or 366.41). People with diabetes were also identified through records of prescriptions for insulin and oral hypoglycemic agents. High-risk patients were those with a diagnosis of peripheral neuropathy, peripheral arterial disease, significant structural abnormalities, prior foot ulcer, or lower limb amputation. These patients comprised $17 \%$ of all persons with diabetes in the registry. A random sample of 230 high risk patients was selected from each of the eight sites.

The survey was administered by mail. A cover letter accompanying the survey provided information regarding the purpose of the study and the importance of the subject's response. Survey nonrespondents received a reminder letter and follow-up survey four weeks after the initial mailing.

\section{Analysis}

We first conducted a descriptive analysis to identify education and self-care behaviors by race. Chi-squared tests were performed using SPSS (SPSS Inc., Chicago, IL) to determine significance between racial categories. Bivariate and multivariate analyses were conducted to identify factors associated with race, while controlling for potentially confounding variables.

\section{Results}

The survey was mailed to a random sample of 1836 subjects in 2000-2001. Of these, 85 (4.6\%) people were deceased. Of the remaining 1751 participants, 856 responded, for a response rate of $48.9 \%$. A further 41 respondents $(2.4 \%)$ had unclear diagnoses, 43 respondents $(2.5 \%)$ mailed incomplete surveys and were dropped from the analysis, and 52 were excluded as they did not indicate their race. The final analysis included 728 surveys, or $41.6 \%$ of the sample.

The average age of the survey participants was 67 years, with $96 \%$ male (data not shown). By race, $81 \%$ were White; 13\%, African American; 4\%, Asian; and 2\%, American Indian and Pacific Islanders (Table 1). Education of 12 years 
Table I Demographic and health history information on survey participants with diabetes and high-risk foot conditions

$\begin{array}{llllll}\text { Total } & \text { White, Hispanic } & \text { African American } & \text { Asian } & \text { American Indian } & \text { p-value } \\ \mathbf{n = 7 1 7} & \mathbf{n}=\mathbf{5 7 9} & n=\mathbf{9 1} & \begin{array}{l}\text { Pacific Islander } \\ n=17\end{array} & \\ & & & & \\ \end{array}$

\begin{tabular}{|c|c|c|c|c|c|c|}
\hline \multicolumn{7}{|l|}{ Demographics } \\
\hline Percent of total & 100 & 81 & 13 & 4 & 2 & \\
\hline Average age, years & 67 & 68 & 63 & 62 & 59 & \\
\hline Education $\leq 12$ years, $\%$ & 63 & 64 & 61 & 48 & 41 & 0.08 \\
\hline Income level $20 \mathrm{~K}$ or less, $\%$ & 79 & 77 & 83 & 93 & 80 & 0.18 \\
\hline Rate general health: fair, poor, \% & 68 & 68 & 65 & 77 & 59 & 0.53 \\
\hline Only VA health insurance, \% & 41 & 38 & 56 & 50 & 35 & $<0.01$ \\
\hline \multicolumn{7}{|l|}{ Smoker (\%) } \\
\hline Current smoker & 24 & 24 & 30 & 20 & 23 & 0.62 \\
\hline Former smoker & 78 & 78 & 80 & 68 & 75 & 0.57 \\
\hline \multicolumn{7}{|l|}{ Health history (\%) } \\
\hline Depression & 34 & 32 & 33 & 47 & 65 & 0.02 \\
\hline Sores/ulcers on feet/legs & 22 & 21 & 22 & 22 & 29 & 0.89 \\
\hline Surgery to fix vessels, feet or legs & 13 & 13 & 12 & 6 & 12 & 0.69 \\
\hline History of prior amputation & 13 & 14 & 14 & 9 & 13 & 0.99 \\
\hline
\end{tabular}

or less was reported by $63 \%$ of the sample, with White/ Hispanic and African Americans reporting the lowest education levels. An annual income of less than $\$ 20,000$ was reported by $79 \%$ of the sample. Asians reported lower income levels and only fair or poor health more often than Whites. African Americans were more likely to have only VA care, and more likely to be current smokers than individuals in other racial groups. American Indians and Pacific Islanders reported significantly higher rates of depression than the other groups.

The majority of respondents felt like they did not "know enough" about various footcare practices (Table 2). In only three categories, "check feet regularly", "keep feet clean" and "wearing shoes always" $\geq 50 \%$ of respondents reported they "know enough". Whites and American Indian/Pacific Islanders were more likely to report knowing enough about foot care self-management, while Asians and African Americans more often reported knowing nothing. Table 3 shows the frequency of select self-care practices by barriers to good foot care and race.

Reported foot hygiene practices were highest in Asians. Barriers to good foot care were reported by Asians in every category. American Indians/Pacific Islanders indicated they uniformly knew what to do and how to do it.

An interesting trend emerges in Table 4 linking the education reported by diabetic veterans who "knew enough" to their current foot care behavior. Of those who "knew enough" about checking feet regularly, one third of Whites and more than half of African Americans never looked at the soles of their feet. Always wearing protective footwear indoors is a priority for patients with diabetes, yet among veterans who "knew enough" about this topic, 19\%-56\% walked barefoot indoors in the last month. The trend continues with avoidance of extreme water temperatures when bathing (a source of foot injury/burns) with $60 \%$ of American Indian/Pacific Islanders and 39\% of Whites reporting "never" testing water temperature, even though half of each group felt they "knew enough" on the topic. African Americans showed keeping their skin moist was a priority as every respondent reported moisturizing their feet at least weekly (data not shown), and nearly half reported using moisturizers every day (Table 3 ). Only 36\% felt they "knew enough" on this topic.

\section{Discussion}

This study illustrates important gaps between self-reported knowledge and foot care practices, highlighting differences by race. White and American Indian/Pacific Islanders generally felt they knew enough, but also had the highest frequency of suboptimal foot care self-management. White veterans generally reported fewer barriers to self-care, thus the observed disconnect between education and behavior could not be attributed solely to contextual barriers. For example, $60 \%$ of Whites knew enough about always wearing shoes, but they were also the most likely to walk barefoot inside. Asians and American Indians/Pacific Islanders in the survey identified a 


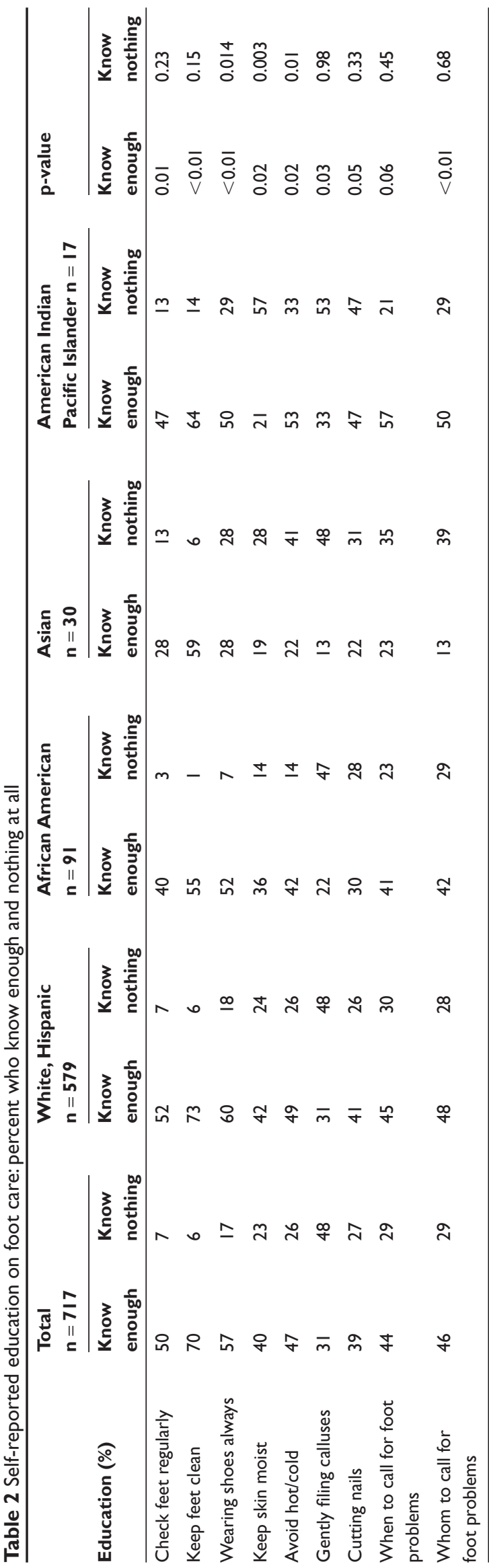

lack of education and resources as major barriers, and were far more likely than their White counterparts to feel they did not know enough on a given topic, indicating an opportunity for enhanced self-management education and care in these groups.

Differences among racial and ethnic groups varied by specific behaviors; ie, American Indians/Pacific Islanders were least likely to inspect their feet daily. Asians were significantly more likely to wash their feet and test water temperatures. These differences reinforce the need to assess self-management skills on an individual basis in order to target priority behaviors.

Differences in reported behavior between ethnic groups may reflect broader underlying cultural differences. For example, Whites generally reported high levels of foot selfcare knowledge but low levels of beneficial behaviors, while Asians reported low levels of foot self-care knowledge, yet had a number of favorable self-care behaviors. Re-framed from a cultural perspective, the question becomes one of reporting, rather than real-world behavior - the difference may be a cultural reluctance to over-report knowledge (in Asians) vs a cultural tendency to over-report (in Whites) rather than true differences in absolute, real-world behavior.

Individualized self-management education is not a new concept. Several publications posit specific steps in foot care education. Self-management education to assist individuals at high risk for foot ulcers and amputation includes (1) assessment and feedback on current selfmanagement behaviors, (2) collaborative setting of specific self-management goals, (3) identification of barriers and social environmental supports to accomplish goals, (4) developing individually tailored problem-solving strategies to overcome obstacles and (5) including strategies for follow-up support. ${ }^{21}$ Our study highlights the importance of understanding racial differences when attempting to create a personalized self-management plan.

Prior studies have shown that broadly targeted clinical strategies are less effective than specific interventions in reducing disparities in care along racial ethnic lines. ${ }^{22}$ This supports self-management steps presented above from Glasgow and colleagues ${ }^{21}$ focusing on highly specific diabetes foot care self-management interventions.

The major strength of this study was the use of a validated foot care specific survey in a large, multi-site sample of individuals with racial and ethnic diversity, in the largest health care system in the USA. A limitation in this study is the way race was coded on the original survey. 
Table 3 Self care practices and barriers to foot care in eight-site study

$\begin{array}{llllll}\text { Total } & \text { White, Hispanic } & \text { African American } & \text { Asian } & \text { American Indian } & \text { p-value } \\ n=717 & n=579 & n=91 & n=30 & \begin{array}{l}\text { Pacific Islander } \\ n=17\end{array}\end{array}$

\begin{tabular}{|c|c|c|c|c|c|c|}
\hline \multicolumn{7}{|l|}{ Self care practices (\% Daily) } \\
\hline Looked bottom feet & 32 & 31 & 35 & 38 & 24 & 0.66 \\
\hline Washed feet & 58 & 56 & 64 & 81 & 59 & 0.02 \\
\hline Soaked feet for $10 \mathrm{~min}$ & 10 & 10 & 8 & 16 & 6 & 0.58 \\
\hline Tested water temperature & 34 & 32 & 40 & 42 & 35 & 0.42 \\
\hline Used lubricants & 24 & 19 & 49 & 34 & 18 & $<0.01$ \\
\hline Filed calluses & 3 & 2 & 6 & 7 & 6 & 0.07 \\
\hline Checked shoes & 33 & 32 & 43 & 38 & 31 & 0.18 \\
\hline Changed shoes & 23 & 23 & 21 & 29 & 13 & 0.63 \\
\hline Walked barefoot inside & 41 & 45 & 28 & 19 & 13 & $<0.01$ \\
\hline \multicolumn{7}{|l|}{ Barriers to good foot care (\%) } \\
\hline I couldn't see well enough & 19 & 18 & 16 & 34 & 18 & 0.13 \\
\hline I couldn't reach my feet & 38 & 38 & 37 & 47 & 41 & 0.76 \\
\hline I couldn't afford it & 9 & 8 & 6 & 31 & 7 & $<0.01$ \\
\hline I didn't think it was important & 7 & 7 & 1 & 25 & 6 & $<0.01$ \\
\hline I didn't know what to do & 10 & 9 & 12 & 19 & 12 & 0.32 \\
\hline $\begin{array}{l}\text { I knew what to do, but not how } \\
\text { to do it }\end{array}$ & 10 & 9 & 17 & 19 & 0 & 0.01 \\
\hline
\end{tabular}

As the prevalence of diabetes continues to increase, ${ }^{1}$ foot care for people with diabetes will be an increasingly important area of both research and clinical care. When looking at sequelae of diabetes, foot care is particularly important, since many lower limb complications may be reduced or even prevented given development and reinforced self-management skills. ${ }^{23}$ Another limitation is inherent in study design - survey nonrespondents may represent those veterans with the lowest levels of confidence and knowledge regarding foot self-care, and our data would then represent an overestimate of actual foot self-care knowledge among veterans with diabetes.

\section{Acknowledgments}

The authors wish to thank VA Health Services Research and Development and Rehabilitation Research and Development for their support. The authors report no conflicts of interest in this work.

Table 4 Relationship between education and self-care in eight-site study of veterans who knew enough about select foot care topics

\begin{tabular}{|c|c|c|c|c|c|}
\hline Foot Care Topic Education (\%) $\rightarrow \leftarrow$ Self-Care (\%) & $\begin{array}{l}\text { White, } \\
\text { Hispanic }\end{array}$ & $\begin{array}{l}\text { African } \\
\text { American }\end{array}$ & Asian & $\begin{array}{l}\text { American Indian } \\
\text { Pacific Islander }\end{array}$ & p-value \\
\hline $\begin{array}{l}\text { Education: Know enough about checking your feet } \\
\text { regularly... }\end{array}$ & 52 & 40 & 28 & 47 & 0.01 \\
\hline Self-care: Never looked at bottom of my feet & 33 & 55 & 0 & 0 & 0.40 \\
\hline $\begin{array}{l}\text { Education: Know enough about wearing shoes } \\
\text { or slippers all the time... }\end{array}$ & 60 & 52 & 28 & 50 & $<0.01$ \\
\hline $\begin{array}{l}\text { Self-care: Walked barefoot indoors in the past } \\
\text { four weeks }\end{array}$ & 56 & 43 & 19 & 43 & 0.12 \\
\hline $\begin{array}{l}\text { Education: Know enough about avoiding very hot } \\
\text { and very cold temperatures to your feet... }\end{array}$ & 49 & 42 & 22 & 53 & 0.02 \\
\hline Self-care: Never tested water temperature & 39 & 25 & 8 & 60 & 0.13 \\
\hline $\begin{array}{l}\text { Education: Know enough about how to keep } \\
\text { skin moist... }\end{array}$ & 42 & 36 & 19 & 21 & 0.02 \\
\hline Self-care: Never used lubricants & 31 & 0 & 10 & 20 & $<0.01$ \\
\hline
\end{tabular}




\section{References}

1. Centers for Disease Control and Prevention. Number of people with diabetes increases to 24 million. Jun 24, 2008. Cited Aug 14, 2008. Available from: http://www.cdc.gov/media/pressrel/2008/ r080624.htm.

2. Miller DR, Safford MM, Pogach LM. Who has diabetes? Best estimates of diabetes prevalence in the Department of Veterans Affairs based on computerized patient data. Diabetes Care. 2004;27(Suppl 2):B10-21.

3. Reiber GE, Koepsell TD, Maynard C, Haas LB, Boyko EJ. Diabetes in nonveterans, veterans, and veterans receiving Department of Veterans Affairs health care. Diabetes Care. 2004;27(Suppl 2):B3-9.

4. Boyko EJ, Ahroni JH, Stensel V, Forsberg RC, Davignon DR, Smith DG. A prospective study of risk factors for diabetic foot ulcer. The Seattle Diabetic Foot Study. Diabetes Care. 1999;22:1036-1042.

5. Boyko EJ, Ahroni JH, Cohen V, Nelson KM, Heagerty PJ. Prediction of diabetic foot ulcer occurrence using commonly available clinical information: the Seattle Diabetic Foot Study. Diabetes Care. 2006;29:1202-1207.

6. Heisler M, Smith DM, Hayward RA, Krein SL, Kerr EA. Racial disparities in diabetes care processes, outcomes, and treatment intensity. Med Care. 2003;41:1221-1232.

7. Johnston MV, Pogach L, Rajan M, et al. Personal and treatment factors associated with foot self-care among veterans with diabetes. $J$ Rehabil Res Dev. 2006;43:227-238.

8. Norris S, Engelgau MM, Venkay Narayan KM. Effectiveness of self-management training in type 2 diabetes. A systematic review of randomized controlled trials. Diabetes Care. 2001;24:561-587.

9. Fitzgerald JT, Gruppen LD, Anderson RM, Funnell MM, Jacober SJ, Grunberger G, Aman LC. The influence of treatment modality and ethnicity on attitudes in type 2 diabetes. Diabetes Care. 2000;23:313-318

10. Wing RR, Goldstein MG, Acton KJ, et al. Behavioral science research in diabetes: lifestyle changes related to obesity, eating behavior, and physical activity. Diabetes Care. 2001;24:117-123.

11. Mensing C, Boucher J, Cypress M, et al. National standards for diabetes self-management education. Diabetes Care. 2006;29(Suppl 1): S78-85.
12. van Dam HA, van der Horst F, van den Borne B, et al. Provider-patient interaction in diabetes care: effects on patient self-care and outcomes. A systematic review. Patient Educ Couns. 2003;51:17-28.

13. Lorig KR, Holman H. Self-management education: history, definition, outcomes, and mechanisms. Ann Behav Med. 2003;26:1-7.

14. Anderson RM. Patient empowerment and the traditional medical model. A case of irreconcilable differences? Diabetes Care. 1995;18:412-415.

15. Jones H, Edwards L, Vallis TM, et al. Changes in diabetes self-care behaviors make a difference in glycemic control: The Diabetes Stages of Change (DiSC) study. Diabetes Care. 2003;26:732-727.

16. Nwasuruba C, Khan M, Egede LE. Racial/ethnic differences in multiple self-care behaviors in adults with diabetes. J Gen Intern Med. 2007;22:115-120.

17. Chin MH, Polonsky TS, Thomas VD, Nerney MP. Developing a conceptual framework for understanding illness and attitudes in older, urban African Americans with diabetes. Diabetes Educ. 2000;26:439-449.

18. Wrobel JS, Charns MP, Diehr P, et al. The relationship between provider coordination and diabetes-related foot outcomes. Diabetes Care. 2003;26:3042-3047.

19. Stewart AL, Hays RD, Wells KB, Rogers WH, Spritzer KL, Greenfield $\mathrm{S}$. Long-term functioning and well-being outcomes associated with physical activity and exercise in patients with chronic conditions in the Medical Outcomes Study. J Clin Epidemiol. 1994;47:719-730.

20. Kaplan S. Diabetes Quality Improvement Project: Patient Survey Final Report: Report to National Committee for Quality Assurance. Washington, DC: National Committee for Quality Assurance;2001.

21. Glasgow RE, Hiss RG, Anderson RM, et al. Report of the health care delivery work group: behavioral research related to the establishment of a chronic disease model for diabetes care. Diabetes Care. 2001;24:124-130.

22. Duru OK, Mangione CM, Steers NW, et al. The association between clinical care strategies and the attenuation of racial/ethnic disparities in diabetes care: the Translating Research Into Action for Diabetes (TRIAD) Study. Med Care. 2006;44:1121-1128.

23. Singh N, Armstrong DG, Lipsky BA. Preventing foot ulcers in patients with diabetes. JAMA. 2005;293:217-228. 